\title{
RNF2 interacts with the linker region of the human P-glycoprotein
}

\author{
PREMA S. RAO ${ }^{1}$, KAVITA B. MALLYA ${ }^{2}$, KALKUNTE S. SRIVENUGOPAL ${ }^{1}$, \\ K.C. BALAJI ${ }^{3}$ and U. SUBRAHMANYESWARA RAO ${ }^{1}$

\begin{abstract}
${ }^{1}$ The Anticancer Resistance Research Group and the Center for Cancer Biology, Department of Pharmaceutical Sciences, Texas Tech University Health Sciences Center, Amarillo, TX 79106; Departments of ${ }^{2}$ Biochemistry and Molecular Biology and ${ }^{3}$ Urology, University of Nebraska Medical Center, Omaha, NE 68198, USA
\end{abstract}

Received July 11, 2006; Accepted August 21, 2006

\begin{abstract}
The human P-glycoprotein (Pgp) is a drug-efflux pump responsible for innate or acquired multidrug resistance in many cancers. Pgp contains a unique $\sim 75$ amino acid long linker region in its middle, which is critically important for its drug transport and ATPase functions. To identify cellular proteins that bind to this linker region and modulate Pgp function, a yeast two-hybrid analysis was carried out. This procedure identified RNF2 (RING finger protein 2), an E3 ubiquitin ligase, as a prominent Pgp-interacting protein. Coexpression of RNF2 with Pgp in Sf9 insect cells resulted in decreased ATPase activity and proteolytic protection of the transporter protein. Immunoprecipitation experiments confirmed the physical interaction between these two proteins. Confocal microscopy showed the presence of RNF2 in the cytoplasm of the Pgp-negative, drug-sensitive MCF-7 breast cancer cells. However, it was undetectable in the Pgp-positive and drug-resistant MCF-7 cells. We suggest that RNF2 regulates the cellular abundance of Pgp, and plays a key role in the development of cancer drug resistance through its own down-regulation.
\end{abstract}

\section{Introduction}

Cancers undergoing chemotherapy often develop resistance to a wide variety of anticancer drugs; this phenomenon is

Correspondence to: Dr U.S. Rao, Department of Pharmaceutical Sciences, Texas Tech University Health Sciences Center, 1300 S. Coulter St. Amarillo, TX 79106, USA

E-mail: us.rao@ttuhsc.edu

Abbreviations: MDR, multidrug resistance; Pgp, P-glycoprotein; RING, really interesting new gene; PCR, polymerase chain reaction; RT-PCR, reverse transcription-PCR; BV, baculovirus; PBS, phosphate-buffered saline

Key words: cancer drug resistance, MDR, P-glycoprotein, ATPase, yeast two hybrid, interactor, RNF2, ubiquitin known as multidrug resistance (MDR). While detoxification of drugs, alterations in the cellular targets for anticancer drugs and pro-apoptotic pathways equally manifest the drug resistance phenomenon, active anticancer drug efflux by the members of a large family of proteins commonly known as ABC-transporters has been well-established as the most common mechanism of MDR in cancer cells (1). Pgp, an $M D R 1$ gene product and a well-characterized member of the above family, is a plasma membrane bound active transporter, which extrudes many anticancer drugs in an energy-dependent manner $(2,3)$. Human Pgp is a 1280 amino acid long polypeptide, which folds into two distinct $\mathrm{NH}_{2}$-half and $\mathrm{COOH}$ half domains. Each of these is $\sim 600$ amino acids long and contains six transmembrane segments and an ATP binding domain. A unique and highly hydrophilic stretch of $\sim 75$ amino acids (residues 633-709), known as the linker region lies in between the above two halves of Pgp $(4,5)$. Studies also have shown that the linker region plays a critical role in the drug efflux and ATPase functions of Pgp $(5,6)$, which is thus likened to the R-domain of the cystic fibrosis transmembrane conductance regulator. Our recent biochemical studies have suggested that the linker region in the Pgp molecule is highly susceptible for breakage and well-accessible to different proteases $(7,8)$, indicating that this exposed region is likely to be a site for protein-protein interactions in vivo. Since Pgp is potentially capable of extruding important cellular constituents leading to cell death, we hypothesized that such an undesirable Pgp function is prevented by cellular regulatory proteins by direct binding to the linker region of the transporter. We tested this hypothesis by yeast two-hybrid analysis, and identified RNF2 as an interacting protein with the linker region of Pgp. Characterization of RNF2-Pgp interactions are reported in this article.

\section{Materials and methods}

Materials. Alexa Fluor-coupled antibodies and anti-hexahistidine antibodies were obtained from Invitrogen, CA. SDS-PAGE and Western blotting reagents were purchased from Bio-Rad. All other reagents used were of analytical grade. 
Molecular biological methods. Standard recombinant DNA procedures of plasmid DNA preparation, subcloning into vectors were used.

Yeast two-hybrid screening. The pGBKT7 and pGADT7 vectors, yeast strains AH109 and Y187 and the procedures of yeast two-hybrid analysis were derived from the Matchmaker Two-Hybrid System 3 kit (Clontech, CA). The human prostate adenocarcinoma cDNA library subcloned into the pGADT7 vector and transformed into the haploid yeast AH109 strain was described previously (9). The cDNA encoding the linker region of Pgp (amino acids 633-707) was subcloned into the pGBKT7 vector and transformed into the yeast strain Y187 and generated the bait strain. The bait was mated with the human prostate adenocarcinoma cDNA library in the AH109 strain. The diploid yeast colonies resulting from this mating that exhibited both growth on quadruple dropout (trp-, leu-, ade- and his-) SD minimal agar medium and B-galactosidase activity as determined by the hydrolysis of $\mathrm{X}-\alpha$-Gal (5-bromo4-chloro-3-inoyl- $\alpha$-D-galactopyranoside) (Clontech, CA) were subjected to further evaluation. The pGADT7 library plasmids from the positive clones were isolated and sequenced at a commercial DNA sequencing facility. The identity of the insert DNA sequences was determined by using the NCBI BLAST server. Tests involving empty vector and an unrelated protein fragment (the cytoplasmically located $\mathrm{NH}_{2}$-terminus of the $\alpha-\mathrm{ENaC}$ subunit) were conducted to eliminate spurious interactions according to the manufacturer's instructions.

Cell lines and culture conditions. Drug-sensitive MCF-7, adriamycin-resistant $\mathrm{MCF}-7 / \mathrm{Adr}^{\mathrm{R}}$ and mitoxantrone-resistant MCF-7/Mito ${ }^{\mathrm{R}}$ cells were kindly donated by Dr Kenneth Cowan (10). The cells were maintained in Dulbecco's modified Eagle's medium, supplemented with $10 \%(\mathrm{v} / \mathrm{v})$ fetal bovine serum and antibiotics (penicillin, $100 \mathrm{U} / \mathrm{ml}$; and streptomycin, $100 \mu \mathrm{g} / \mathrm{ml}$ ). Cells were grown in a $37^{\circ} \mathrm{C}$ humidified incubator with $5 \% \mathrm{CO}_{2}$.

Reverse transcription-PCR (RT-PCR). Isolation of total RNA, reverse transcription and RT-PCR have been described previously (11). Amplification of RNF2 was carried out using forward primer 5'-GGAGCAGAAGATAATGGTGACAG-3' and reverse primer 5'-GTGCTCCTTTGTAGGTGCGTAAT-3. The number of PCR cycles was 30 .

Isolation of full-length RNF2 cDNA. The 1.1-kb RNF2 cDNA was isolated from the total RNA of prostate PC3 as well as MCF-7 cells by the previously described RT-PCR procedure (11) using the $R N F 2$-specific primer pairs, 5'-GCCGAATTC ATGTCTCAGGCTGTGCAGA-3', and 5'-GCCGGATCCTC ATTTGTGCTCCTTTGTA-3'. A cDNA sequence coding for 6-His tag, HHHHHHM was added just before the stop codon at its 3'-end to express RNF2-6His. This cDNA was subcloned into the pVL1392 baculoviral transfer vector.

Preparation of recombinant $B V$ and estimation of Pgp ATPase activity. The above isolated $R N F 2$ cDNA and metallothionein2a cDNA (control) were inserted into pVL1392 baculoviral transfer vectors (Pharmingen, CA). The RNF2-BV and the control Met2a-BV were prepared using the above baculoviral transfer vectors and BaculoGold DNA (BD Pharmingen, CA)
MSQAVQTNGTQPLSKTWELSLYELQRTPQEAITDGLE IVVSPRSLHSE LMCP ICLDMLKNTMTTKECLLHRFCADCI ITALRSGNKE CPTCRKKLVS KRSLRPDPNFDALI SKIYPSRDEYEAHQERVLARINKHNNQQALSHSI EEGLKIQAMNRLQRGKKQQIENGSGAEDNGDSSHCSNASTHSNQEAGE SNKRTKT SDDSGLELDNNNAAMA IDPVMDGASE IELVFRPHPTLMEKD DSAQTRY IKTSGNATVDHLSKYLAVRLALEELRSKGESNOMNLDTASE KOYTIYIATASGQFTVLNGSFSLELVSEKYWKVNKPMELYYAPTKEHK

Figure 1. Characteristics of RNF2. The deduced amino acid sequence from the RNF2 cDNA isolated from MCF-7 cells. The RING finger domain is italicized and the Cys and His residues in this domain are underlined. The putative nuclear localization sequences are indicated in boxes. The region of RNF2 that was found to interact with the linker region is double-underlined.

as reported previously (12). Preparation of the human Pgpexpressing MDR1-BV has been reported (12). The Sf9 insect cells were infected with these recombinant BVs, the total membrane fraction was prepared and the drug-stimulated Pgp ATPase activity was determined as described previously (12).

Preparation of anti-RNF2. To raise RNF2-specific antibodies, the amino acid sequence, 231-RPHPTLMEKDDSAQTR YIK-249 in the RNF2 molecule was used as an epitope. Synthesis of this immunizing peptide and coupling to carrier protein, keyhole limpet hemacyanin and generation of antibodies in rabbit were carried out at a commercial location (AnimalPharm Services, CA). Immunoblotting was used to screen for the presence of antibodies in the test bleed sera and the antibody was termed anti-RNF2. The antibody was affinity purified using Sulfolink-Agarose beads (Pierce, IL) as described by the manufacturer's instructions.

Confocal microscopy. To visualize RNF2 and Pgp expression, cells growing on coverslips were fixed with an ice-cold acetone: methanol (1:1) mixture for $5 \mathrm{~min}$. Cells were then stained with primary antibody [anti-RNF2 or anti-Pgp $\left(\mathrm{NH}_{2} 11\right)$ followed by staining with the Alexa Fluor 488-coupled appropriate secondary antibody (Molecular Probes, Eugene, OR] as described previously (11). Fluorescence in cells was visualized by using a Leica laser confocal microscope (TCS SL; Leica Microsystems, Heidelberg, $\mathrm{GmbH}$ ) and the images were digitally recorded on a computer hard drive.

Immunohistochemistry. RNF2 and Pgp in paraffin-embedded human breast adenocarcinomas were immunostained using anti-RNF2 and $\mathrm{NH}_{2} 11$ antibodies in conjunction with Alexa Fluor 488-conjugated secondary antibodies according to the procedures described previously (11).

Immunoprecipitation and immunoblotting. The Sf9 insect cells were infected with the MDR1-BV and RNF2-BV or Met2a-BV at a ratio of 1:8 (plaque forming units) for 2 days and the cells were lysed in RIPA buffer (PBS containing 1\% NP40, $0.5 \%$ sodium deoxycholate, $0.1 \%$ SDS, and protease inhibitors (Roche Diagnostics, Mannheim, Germany) or in a buffer (20 mM MOPS, $2 \mathrm{mM}$ EGTA, $2 \mathrm{mM}$ EDTA, $2 \mathrm{mM}$ orthovanadate, $30 \mathrm{mM} \mathrm{NaCl}(\mathrm{pH} 7.0)$ containing either $1 \%$ NP40, $1 \%$ Triton X-100, or $1 \%$ octyl- $\beta$-glucoside. The insoluble material was removed by centrifugation $(10,000 \mathrm{x} \mathrm{g}$, $15 \mathrm{~min})$. Pgp and RNF2 proteins present in the supernatants were immunoprecipitated using $\mathrm{NH}_{2} 11$ and anti-RNF2 anti- 

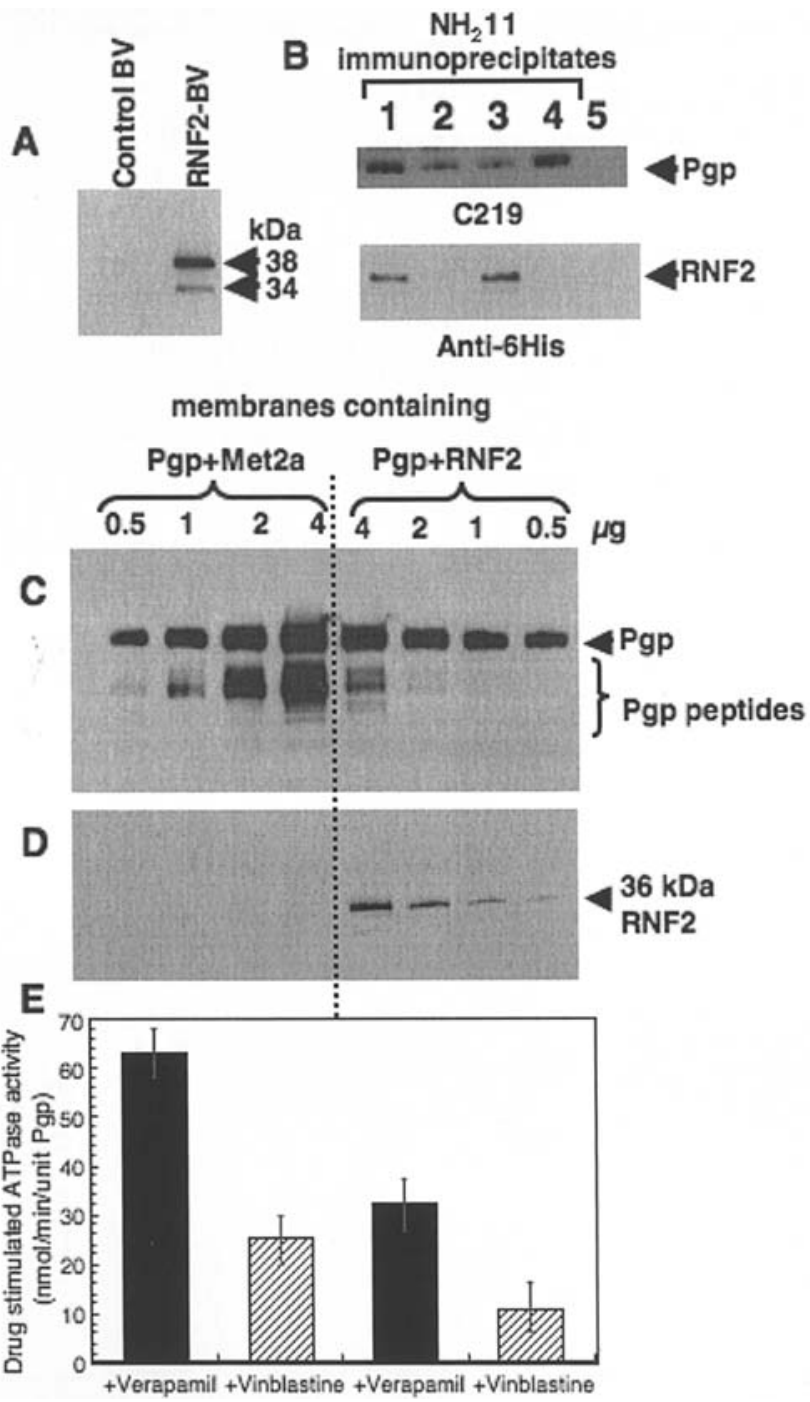

Figure 2. Characterization of RNF2. Interaction with Pgp. A, immunoblotting analysis of Sf9 insect cells infected with Met2a-BV (control BV) and RNF2BV using anti-6His antibody. The $~ 38$ - and 34-kDa proteins are full-length and truncated RNF2. B, co-immunoprecipitation of RNF2 with Pgp. The RNF2 and Pgp expressing Sf9 insect cells were lysed in buffers containing 1\% NP-40 (lane 1 ), $1 \%$ octyl- $\beta$-glucoside (lane 2), $1 \%$ Triton X-100 (lane 3) or in RIPA buffer (lane 4). Pgp was immunoprecipitated with $\mathrm{NH}_{2} 11$. The Triton $\mathrm{X}-100$ soluble material was immunoprecipitated with pre-immune serum (lane 5). The immunoprecipitates were analyzed by immunoblotting using the $\mathrm{C} 219$ (panel: C219) and anti-6His (panel: anti-6His) to detect Pgp and RNF2. C, effect of RNF2 on the endogenous degradation of Pgp. The Sf9 insect cells infected with MDR1 BV with either Met2a-BV or RNF2-BV and the membrane fractions were prepared. Different amounts of total membrane protein were analyzed by immunoblotting using $\mathrm{NH}_{2} 11$ to detect Pgp. Pgp and its peptides were indicated. A duplicate immunoblot was developed with anti-RNF2 to detect RNF2 (D). E, effect of RNF2 on the Pgp ATPase activity. The membrane fractions were assayed for the Pgp ATPase activity in the presence of $50 \mu \mathrm{M}$ verapamil and $10 \mu \mathrm{M}$ vinblastine, as described previously (12).

bodies respectively, and the proteins were separated on SDS-polyacrylamide gels, followed by electrotransfer onto PVDF membranes as described previously (7). The blots were developed using either the $\mathrm{NH}_{2} 11 / \mathrm{C} 219$ (Pgp-recognizing antibodies), anti-His antibody (to recognize the 6-His tag in the RNF2-6His protein) or anti-RNF2 antibody in conjunction with horseradish peroxidase-coupled secondary antibody and the enhanced chemiluminescence assay kit (Amersham, Piscataway, NJ) as described previously (7).
Protein estimation. The protein content in the samples was estimated by the Bio-Rad DC protein assay kit using bovine serum albumin as standard.

\section{Results}

Identification of RNF2 as an interacting protein with the linker region of Pgp. To identify proteins that interact with the linker region of Pgp, we performed the yeast two-hybrid analysis using the linker region of Pgp as bait and the human prostate adenocarcinoma cDNA library as source of interacting proteins, as described in Materials and methods. This screen identified multiple copies of four different recognizable clones by a homology search in the GenBank database. These four clones contained coding sequences from RNF2 (accession no. BC012583), C35 protein (accession no. AY508814), serine and arginine rich protein (accession no. AF314185) and mitochondrial flavin protein (accession no. BC029995). The library plasmids from these clones were subsequently retransformed into yeast carrying the pGBKT7/linker region, pGBKT7/ $\mathrm{NH}_{2}$-terminus of the $\alpha$ subunit of ENaC or pGBKT7 vector, and plated on quadruple drop-out $\mathrm{SD}$ minimal agar plates $\left(\mathrm{Leu}^{-}, \mathrm{Trp}^{-}, \mathrm{Ade}^{-}\right.$and $\mathrm{His}^{-}$) and $\alpha-\mathrm{X}-\mathrm{Gal}$. The results of this study indicated that only the RNF2 cDNA is a true positive interactor with the linker region. This entire yeast two-hybrid analysis was repeated twice with identical results. Additionally, we performed the yeast two-hybrid analysis using several different bait sequences including the $\mathrm{NH}_{2}$-terminus of Pgp, $\mathrm{NH}_{2}$-terminus of the $\alpha$-ENaC subunit, $\mathrm{COOH}$-terminus of the $\alpha-\mathrm{ENaC}$ subunit, full-length protein kinase $\mathrm{C} \mu$, kinase domain of the protein kinase $\mathrm{C} \mu$, pleckstrin homology domain of protein kinase $\mathrm{C} \mu$, and a domain of the human transglutaminase, using the identical prey library. In all, more than 150 clones were isolated and sequenced, and none of them were found to code for RNF2 (data not shown). Collectively, these observations suggested that the yeast two-hybrid analysis procedure employed in this study was specific and identified RNF2 as a unique interacting protein of the linker region of Pgp. Fig. 1 shows the full-length amino acid sequence of RNF2 highlighting the various functional domains. RNF2 is a 336-amino acid long polypeptide and contains a characteristic C3HC4 RING finger motif with 7 cysteines and a histidine in between residues 51 and 90 (13). RNF2 also contains two putative nuclear localization sequences, 88-PTCRKKL-94 and 192-PSNKRTK-198. Importantly, the RNF2 cDNA that was present in the yeast pGADT7 library vector codes for the residues 176-336 in the RNF2 polypeptide, suggesting that the C-half of RNF2 constitutes an interacting domain with the linker region of Pgp.

RNF2 expression in Sf9 insect cells. To establish that RNF2-BV allows the expression of a structurally integral RNF2 protein, the Sf9 insect cells were infected with RNF2-BV and the lysates were analyzed by SDS-PAGE followed by immunoblotting using anti-6His antibody. Fig. 2A shows the presence of two proteins with molecular masses of $\sim 38$ and $34 \mathrm{kDa}$ in the RNF2-BV-infected Sf9 insect cell lysates, which were absent in control Met2a-BV (vector)-infected cell lysates. Since the calculated mol.mass of RNF2-6His is $\sim 38 \mathrm{kDa}$, the observed $\sim 38 \mathrm{kDa}$ protein represents the full-length RNF2. 
The $\sim 34 \mathrm{kDa}$ protein appears to be a truncated form of RNF2 resulting from a loss of $\sim 40$ amino acids from its $\mathrm{N}$-terminus.

RNF2 binds to Pgp. To determine if RNF2 interacts with Pgp, RNF2-6His was co-expressed with Pgp in Sf9 insect cells, and then lysed in buffers containing different detergents, NP-40, Triton X-100, octyl- $\beta$-glucoside and deoxycholateSDS mixture (constituents of RIPA buffer), as described in Materials and methods. Pgp in these soluble fractions was then immunoprecipitated using Pgp-specific $\mathrm{NH}_{2} 11$ antibody (11) and analyzed by immunoblotting for the presence of Pgp and RNF2-6His by using C219 and anti-6His antibodies, respectively. The upper panel of Fig. 2B shows the presence of Pgp in immunoprecipitates prepared in all different experimental conditions that varied in the type of detergent, suggesting that the $\mathrm{NH}_{2} 11$ immunoprecipitates Pgp. On the other hand, the anti-6His antibody-reactive $\sim 38 \mathrm{kDa}$ RNF2 was found only in immunoprecipitates obtained using NP-40 and Triton X-100-containing buffers (Fig. 2B, panel: anti-6His). These results suggest that RNF2 interacts with Pgp and the interactions between these two proteins were not affected by the NP-40 and Triton X-100 detergents.

RNF2 protects Pgp from proteolysis in Sf9 insect cells. We often noticed Pgp degradation to some extent when membrane fraction was prepared from Sf9 insect cells infected with MDR1-BV in the absence of protease inhibitors (7). These Pgp-related fragments migrated similarly on SDS-PAGE gels as Pgp peptides generated from cleavage of the linker region by different proteases $(7,8)$, suggesting that proteases in Sf9 insect cells could also cleave the linker region. Fig. 2C (left panel) shows a typical pattern of Pgp fragmentation in membrane fraction prepared in the absence of protease inhibitors from Sf9 insect cells co-infected with Pgp- and Met-2a-expressing BV's. Interestingly, when RNF2 was co-expressed with Pgp in place of Met-2a, Pgp degradation was minimal (Fig. 2C, right panel). When these blots were probed with anti-6His antibody, the $\sim 38-\mathrm{kDa}$ RNF2 and its $\sim 34-\mathrm{kDa}$ truncated form to a lesser extent were detectable (Fig. 2D, right panel). Since predictive algorithms suggest that RNF2 is a soluble protein devoid of transmembrane segments, its presence in the membrane fraction is likely to be due to its association with the membrane-bound Pgp. Thus, these results suggest that RNF2 interacts with the linker region of Pgp and such interactions protect the Pgp from the proteolytic enzymes present in the Sf9 insect cells.

RNF2 inhibits the drug-stimulated Pgp ATPase activity. Pgp expressed in Sf9 insect cells exhibits high ATPase activity in the presence of anticancer drugs and chemosensitizers (14). To determine the effect of RNF2 binding to the Pgp linker region on the ensuing influence on drug-stimulated ATPase activity of the transporter, membranes containing Pgp and Met-2a or RNF2, were prepared and assayed for the verapamil- and vinblastine-stimulated ATPase activities of Pgp as described previously (12). The amount of Pgp in these membranes was quantitated by determining the staining intensity in Western blots by densitometry. The amount of Pgp in RNF2-Pgp expressing membranes was normalized to that of Pgp present in Pgp-Met2a containing membranes. The

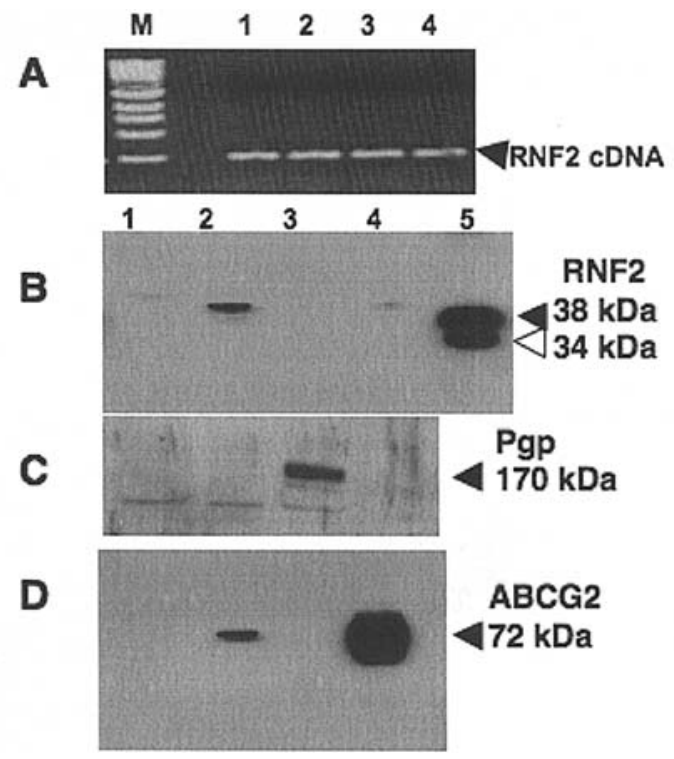

Figure 3. Characterization of RNF2. Expression in different human breast cancer cell lines. A, RT-PCR analysis of RNF2 in different cell lines. Total RNA from MDA-MB231 (lane 1), MCF-7 (lane 2), MCF-7/Adr ${ }^{\mathrm{R}}$ (lane 3) and MCF-7/mitoxantrone ${ }^{\mathrm{R}}$ (lane 4) cells was subjected to RT-PCR using $R N F 2$-specific primers and the reaction mixtures were separated on $1 \%$ agarose gel. The $0.5-\mathrm{kb}$ fragment amplified is the RNF2 cDNA. Standard DNA ladder was included in lane M. B, expression of RNF2 protein. Cell lines indicated in A were analyzed by immunoblotting which was developed with anti-RNF2. RNF2 expressed in Sf9 insect cells was included as a positive control (lane 5). The molecular masses of the RNF2 bands are indicated with arrow heads. $\mathrm{C}$ and $\mathrm{D}$, immunoblots of the same cell lysates using $\mathrm{NH}_{2} 11$ and anti-ABCG2 to detect Pgp and BCRP, respectively.

drug-stimulated ATPase activities are expressed as activity exhibited by unit Pgp. Fig. 2E shows that in the presence of RNF2, the verapamil- and vinblastine-stimulated Pgp ATPase activities were inhibited by nearly $50 \%$ (right panel), when compared to the activity in membranes containing Pgp and Met-2a alone (left panel). Since the linker region is critical for the Pgp ATPase function, reduced drug-stimulated ATPase activity is likely due to the binding of RNF2 to this critical region of the transporter. The observations that RNF2 coexpression greatly reduces proteolytic cleavage Pgp in the Sf9 insect cells (Fig. 2C), RNF2 and Pgp co-immunoprecipitate (Fig. 2B) and the RNF2 binding to Pgp results in the reduction of drug-stimulated ATPase activity (Fig. 2E), collectively suggest that RNF2 binds to the linker region leading to an overall decrease in the drug-stimulated ATPase function of Pgp.

RNF2 is transcribed to similar extents in the drug-sensitive and -resistant human breast cancer cells. The panel of human breast cancer cells including the drug-sensitive MCF-7, adriamycinresistant MCF-7/Adr ${ }^{\mathrm{R}}$, aggressive breast cancer MDA-MB-231 and mitoxantrone-resistant $\mathrm{MCF}-7 /$ mitoxantrone $^{\mathrm{R}}$ were analyzed for the presence of RNF2 gene transcripts by RTPCR using forward primer, 5'-GGAGCAGAAGATAATG GTGACAG-3' and reverse primer 5'-GTGCTCCTTTGTAG GTGCGTAAT-3, and the results are shown in Fig. 3A. As expected from the use of the specific primer pair, the RT-PCR amplified 0.5-kb RNF2 cDNA, which was present at similar levels in all of the cell lines tested, suggesting that RNF2 is constitutively transcribed. GAPDH cDNA was amplified to 
A

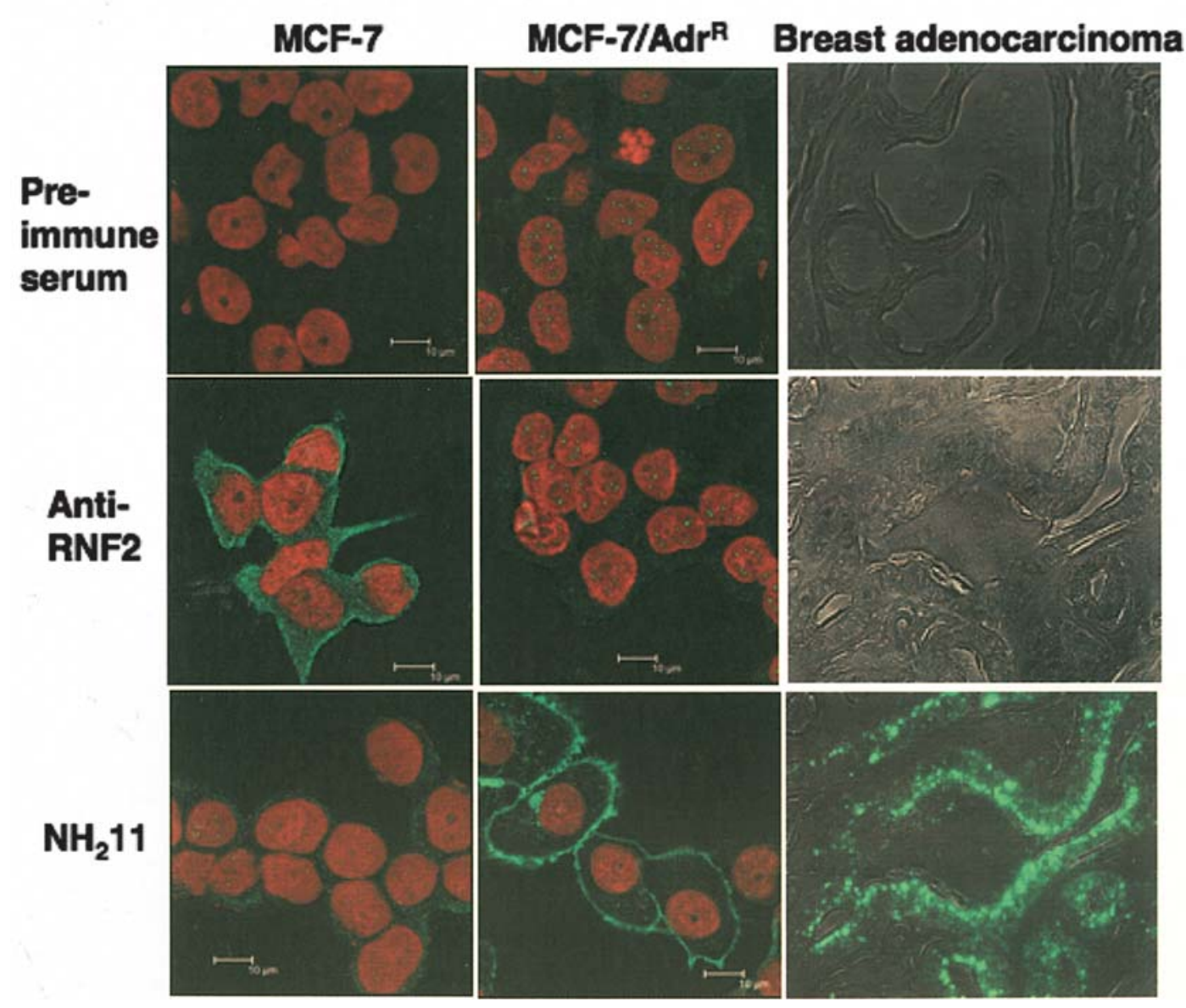

Figure 4. Immunocytochemistry of RNF2 and Pgp. MCF-7 (A) and MCF-7/Adr ${ }^{\mathrm{R}}$ (B) cells were immunostained with preimmune serum (upper panel), antiRNF2 (middle panel) and $\mathrm{NH}_{2} 11$ (lower panel), in conjunction with Alexa Fluor-488-coupled secondary antibody. The nuclei were stained with propidium iodide. The green fluorescence of Alexa Fluor-488 and the red fluorescence of propidium iodide were recorded using a Leica confocal laser microscope. The paraffin-embedded human breast adenocarcinoma sections (C) were also immunostained as above. The Alexa Fluor-488 fluorescence was visualized using a Leica fluorescence microscope.

equal amounts in all these cells, suggesting the level of RNF2 was quantitative (not shown).

RNF2 protein is detectable only in drug-sensitive MCF-7 cells. To determine the levels of endogenous RNF2 protein in breast cancer cell lines, Western blot analysis was carried out and the results are shown in Fig. 3B. A single $\sim 39-\mathrm{kDa}$ protein migrating slightly slower than the $38-\mathrm{kDa}$ RNF2 expressed in Sf9 insect cells was detected in MCF-7 cell lysates. Since RNF2 contains numerous putative phosphorylation sites, it is likely that RNF2 undergoes post-translational modification, which is presently being investigated. However, RNF2 was not detectable in the drug-resistant MCF-7/Adr ${ }^{\mathrm{R}}$ and MCF-7/ mitoxantrone $\mathrm{e}^{\mathrm{R}}$ or in MDA-MB-231 cells. These cell lysates were also analyzed for the presence of Pgp (Fig. 3C) and ABCG2 (Fig. 3D), also known as breast cancer resistance protein (BCRP) by immunoblotting using $\mathrm{NH}_{2} 11$ and antiABCG2 (Chemicon International, Temecula, CA) antibodies. These results, as expected (11), showed that the MCF-7/Adr ${ }^{\mathrm{R}}$ cells express Pgp, whereas, the MCF-7/mitoxantrone ${ }^{\mathrm{R}}$ cells and, MCF-7 cells to a lesser extent, express ABCG2 (15). Although not shown, the MDA-MB-231 cells used in the present study were resistant to methotrexate. Taken together, these data reveal an interesting uncoupling of RNF2 transcription and protein expression. Thus, although all of the tested breast cancer cell lines contained the mRNA for $R N F 2$, only the drug-sensitive MCF-7 cells possessed the anti-RNF2-reactive $\sim 39-\mathrm{kDa}$ protein.

Endogenous RNF2 in MCF-7 cells is predominantly cytoplasmic. To characterize the relationship between RNF2 and $\mathrm{Pgp}$ proteins in drug-sensitive and -resistant cells, $\mathrm{MCF}-7$ and $\mathrm{MCF}-7 / \mathrm{Adr}^{\mathrm{R}}$ cells, were grown on coverslips and immunostained with the anti-RNF2 in conjunction with the Alexa Fluor-488-coupled secondary antibody, and then analyzed by confocal laser microscopy. The cells were stained with pre-immune serum (Fig. 4, upper panels of A-C) to establish the specificity of the RNF2 antibodies. Fig. 4A middle panel shows the fluorescence of Alexa Fluor-488 bound to anti-RNF2, which was present predominantly in the cytoplasm of the drug-sensitive MCF-7 cells. This anti-RNF2 immunostaining was greatly reduced when the antibody premixed with immunizing peptide (not shown). In contrast, the fluorescence intensity associated with anti-RNF2 in the drugresistant MCF-7/Adr ${ }^{\mathrm{R}}$ cells was weak (Fig. 4B, middle panel), which was similar to that of the pre-immune serum (upper 
panel). The nuclei of MCF-7/Adr ${ }^{\mathrm{R}}$ cells stained with anti-RNF2 contained fluorescent speckles, which was also observed with pre-immune serum, indicating that these nuclear speckles were not likely related to RNF2. As a positive control for this immunostaining procedure, cells were immunostained for Pgp with the $\mathrm{NH}_{2} 11$ and the results are shown in Fig. 4 (bottom panel). As expected, the plasma membrane of MCF-7/Adr ${ }^{\mathrm{R}}$ cells but not MCF-7 cells was green fluorescent, suggesting the location of Pgp in the plasma membranes of $\mathrm{MCF}-7 / \mathrm{Adr}^{\mathrm{R}}$ cells.

To further determine the extent of RNF2 expression in Pgp-expressing cells, paraffin-embedded sections from primary specimens of human breast adenocarcinoma were stained for Pgp and RNF2 by using $\mathrm{NH}_{2} 11$ and anti-RNF2, respectively, and the results are shown in Fig. 4C. The Pgp immunostaining was predominantly associated with the luminal side of the ductal epithelial cells of the breast glands. However, the antiRNF2 or the pre-immune serum failed to stain any cells in the sections, including the ductal epithelial cells under these experimental conditions. Thus, the absence of RNF2 staining in cells expressing Pgp is consistent with our data from confocal microscopy. These data demonstrate that Pgp and RNF2 expression is inversely related.

\section{Discussion}

Pgp overexpression in cancers, leading to the development of MDR, continues to be a major stumbling block for successful chemotherapy of human cancers. While an improvement of current anticancer drugs and the development of new and innovative drugs is very necessary, a clear understanding of the biology of tumor cell resistance is also paramount. In this regard, identification of proteins that bind and regulate the human Pgp is highly important for shedding light on causes and reversal of multidrug resistance. As alluded to in the Introduction, Pgp contains a unique $\sim 75$ amino acid long stretch in between the $\mathrm{NH}_{2}$ - and $\mathrm{COOH}$-halves, termed a linker region. This linker region contains several putative phosphorylation and proteolytic sites. While a systematic analysis of the phosphorylation sites in this region was carried out previously $(16,17)$, previous research has demonstrated that the linker region is flexible, and any alteration to this nature of its secondary structure impairs the ability of Pgp to mediate drug-efflux, and consequently, drug resistance (5). In the investigations aimed at understanding the catalytic cycle, we observed that the linker region in the Pgp molecule is most accessible to the interactions with proteases such as trypsin, chymotrypsin and proteinase $\mathrm{K}$ leading to the cleavage of the Pgp molecule into $\mathrm{NH}_{2}$ - and $\mathrm{COOH}$-halves $(7,8)$. In addition, these studies have demonstrated that the linker region is critically important for the coupling of ATPase activity to the drug transport (6). Together, these studies have suggested that the catalytically important linker region is flexible and exposed, and therefore, constitutes the most likely domain of Pgp for possible interactions with cellular proteins.

Our studies identified RNF2 as a specific interactor of the Pgp linker area. RNF2 is a 336 amino acid long polypeptide that contains a unique C3HC4 RING finger motif. A homology search in the GenBank database revealed that this RING finger motif is conserved in a large number of proteins including
BRCA1, RET finger protein (Rft), transcriptional intermediary factor (TIF1), Cbl, Bmi-1, Mel-18 and RING1 (18-27). These RING finger proteins are thought to play roles in the formation and architecture of large protein complexes that contribute to diverse cellular processes such as oncogenesis, apoptosis, development, and protein ubiquitination $(21,28)$. Interestingly, some of these RING finger proteins have the ability to bind DNA and RNA and participate in gene regulation $(24,27,29)$. In addition, many RING finger proteins interact with the polycomb group of proteins and the resulting scaffolding protein complexes that act as transcriptional repressors (29). Recently, RNF2 was reported to be a component of the centromere complex (30), and the presence of two putative nuclear localization sequences we identified in its primary sequence (Fig. 1) could explain it. However, the functional role of RNF2 in the nucleus remains unclear at present.

The results presented in Fig. 4 show that the endogenous RNF2 in MCF-7 cells is predominantly cytoplasmic, suggesting that its function in these cells is probably confined to the cytosol. Interestingly, Kang and co-workers have shown that the C-terminal domain of RNF2 directly interacts with the S6'ATPase (31), a subunit of the cytoplasmically located proteosomal $19 \mathrm{~S}$ regulatory complex, which is a member of the highly conversed AAA (ATPase associated with various cellular activities) protein family (32). Our observations that the C-half of the RNF2 molecule interacts with the linker region and regulates Pgp function raises an important issue that cellular ATPases may interact with RNF2 before undergoing proteosomal degradation.

Our results showed that RNF2 protein is present in the drug-sensitive and Pgp-ve MCF-7 cells. Interestingly, a previous study provides evidence that RNF2 binds to E2 ubiquitin-conjugating enzymes and exhibits E3 ubiquitin ligase activity (33). In addition, Lee and co-workers have demonstrated that RNF2 binds to Hip-2, an E2 ubiquitinconjugating enzyme through its RING finger motif (34). We and others have observed that although the drug-sensitive MCF-7 cells transcribe the MDR1 gene, the Pgp remains undetectable $(11,35)$. Therefore it is tempting to speculate that the nascently translated Pgp in the drug-sensitive MCF-7 cells may serve as a substrate for RNF2-mediated ubiquitination and subsequent proteosomal digestion, thus, contributing to an apparent absence of Pgp in the drug-sensitive cells.

Observations presented in Figs. 3 and 4 indicated that the drug-resistant MCF-7/Adr ${ }^{\mathrm{R}}$ cells do not contain RNF2 protein, even though this gene is well transcribed. Recently, Meek and Knippschild have shown that $\mathrm{mdm} 2 / \mathrm{Hdm} 2$, a protein with RING finger domain drives its own ubiquination followed by rapid degradation (36). The absence of RNF2 in $\mathrm{Pgp}^{+}$cells suggests that RNF2 may similarly undergo selfubiquitination, leading to its apparent absence in these drugresistant cells. Pgp expressed in MCF-7/Adr ${ }^{\mathrm{R}}$ cells and analyzed in our laboratory migrates as a single $\sim 170-\mathrm{kDa}$ protein in immunoblots without signs of a ladder pattern indicative of ubiquitin modification, implying that Pgp-ubiquitination machinery may be impaired in drug-resistant cells. Therefore, an apparent loss of RNF2 may stabilize the Pgp and increase its abundance in multidrug-resistant cancer cells, which is necessary for the survival of these cells during chemotherapy. Studies are underway to test this hypothesis. 


\section{Acknowledgments}

We thank Drs Ulrich Bickel and Parmender Mehta for assistance with immunohistochemical analyses and Ms S. Malaroviyam for the technical assistance. This work was supported by grants from the National Institutes of Health (CA106625), the Department of Defense (BC046411) and the Women's Health Research Institute to U.S. Rao.

\section{References}

1. Annereau JP, Szakacs G, Tucker CJ, Arciello A, Cardarelli C, Collins J, Grissom S, Zeeberg BR, Reinhold W, Weinstein JN, Pommier Y, Paules RS and Gottesman MM: Analysis of ATPbinding cassette transporter expression in drug-selected cell lines by a microarray dedicated to multidrug resistance. Mol Pharmacol 66: 1397-1405, 2004.

2. Ueda K, Cardarelli C, Gottesman MM and Pastan I: Expression of a full-length cDNA for the human 'MDR1' gene confers resistance to colchicine doxorubicin and vinblastine. Proc Natl Acad Sci USA 84: 3004-3008, 1987.

3. Choi KH, Chen CJ, Kriegler M and Roninson IB: An altered pattern of cross-resistance in multidrug-resistant human cells results from spontaneous mutations in the mdr1 (P-glycoprotein) gene. Cell 53: 519-529, 1988.

4. Chen CJ, Chin JE, Ueda K, Clark DP, Pastan I, Gottesman MM and Roninson IB: Internal duplication and homology with bacterial transport proteins in the mdr1 (P-glycoprotein) gene from multidrug-resistant human cells. Cell 47: 381-389, 1986.

5. Hrycyna CA, Airan LE, Germann UA, Ambudkar SV, Pastan I and Gottesman MM: Structural flexibility of the linker region of human P-glycoprotein permits ATP hydrolysis and drug transport. Biochemistry 37: 13660-13673, 1998.

6. Rao US and Nuti SL: Identification of two different states of P-glycoprotein in its catalytic cycle: role of the linker region in the transition between these two states. J Biol Chem 278: 46576-46582, 2003.

7. Nuti SL, Mehdi A and Rao US: Activation of the human Pglycoprotein ATPase by trypsin. Biochemistry 39: 3424-3432, 2000.

8. Nuti SL and Rao US: Proteolytic cleavage of the linker region of the human P-glycoprotein modulates Its ATPase function. J Biol Chem 277: 29417-29423, 2002.

9. Rao PS, Jaggi M, Smith DJ, Hemstreet GP and Balaji KC: Metallothionein 2A interacts with the kinase domain of PKCmu in prostate cancer. Biochem Biophys Res Commun 310: 1032-1038, 2003.

10. Fairchild CR, Moscow JA, O'Brien EE and Cowan KH: Multidrug resistance in cells transfected with human genes encoding a variant P-glycoprotein and glutathione S-transferase-pi. Mol Pharmacol 37: 801-809, 1990.

11. Rao PS, Govindarajan R, Mallya KB, West W and Rao US: Characterization of a new antibody raised against the $\mathrm{NH} 2$ terminus of P-glycoprotein. Clin Cancer Res 11: 5833-5839, 2005.

12. Rao US: Mutation of glycine 185 to valine alters the ATPase function of the human P-glycoprotein expressed in Sf9 cells. J Biol Chem 270: 6686-6690, 1995.

13. Lovering R, Hanson IM, Borden KL, Martin S, O'Reilly NJ Evan GI, Rahman D, Pappin DJ, Trowsdale J and Freemont PS: Identification and preliminary characterization of a protein motif related to the zinc finger. Proc Natl Acad Sci USA 90: 2112-2116, 1993

14. Sarkadi B, Price EM, Boucher RC, Germann UA and Scarborough GA: Expression of the human multidrug resistance cDNA in insect cells generates a high activity drug-stimulated membrane ATPase. J Biol Chem 267: 4854-4858, 1992.

15. Litman T, Jensen U, Hansen A, Covitz KM, Zhan Z, Fetsch P, Abati A, Hansen PR, Horn T, Skovsgaard T and Bates SE: Use of peptide antibodies to probe for the mitoxantrone resistanceassociated protein $\mathrm{MXR} / \mathrm{BCRP} / \mathrm{ABCP} / \mathrm{ABCG} 2$. Biochim Biophys Acta 1565: 6-16, 2002 .

16. Szabo K, Bakos E, Welker E, Muller M, Goodfellow HR, Higgins CF, Varadi A and Sarkadi B: Phosphorylation site mutations in the human multidrug transporter modulate its drugstimulated ATPase activity. J Biol Chem 272: 23165-23171, 1997.
17. Germann UA, Chambers TC, Ambudkar SV, Licht T, Cardarelli CO, Pastan I and Gottesman MM: Characterization of phosphorylation-defective mutants of human P-glycoprotein expressed in mammalian cells. J Biol Chem 271: 1708-1716, 1996.

18. Akasaka T, Tsuji K, Kawahira H, Kanno M, Harigaya K, Hu L, Ebihara Y, Nakahata T, Tetsu O, Taniguchi M and Koseki H: The role of mel-18, a mammalian Polycomb group gene, during IL-7-dependent proliferation of lymphocyte precursors. Immunity 7: 135-146, 1997

19. Barlow PN, Luisi B, Milner A, Elliott M and Everett R: Structure of the $\mathrm{C} 3 \mathrm{HC} 4$ domain by $1 \mathrm{H}$-nuclear magnetic resonance spectroscopy: A new structural class of zinc-finger. J Mol Biol 237: 201-211, 1994.

20. Bienstock RJ, Darden T, Wiseman R, Pedersen L and Barrett JC: Molecular modeling of the amino-terminal zinc ring domain of BRCA1. Cancer Res 56: 2539-2545, 1996.

21. Borden KL: RING domains: master builders of molecular scaffolds? J Mol Biol 295: 1103-1112, 2000.

22. Cao T, Shannon M, Handel MA and Etkin LD: Mouse ret finger protein (rfp) proto-oncogene is expressed at specific stages of mouse spermatogenesis. Dev Genet 19: 309-320, 1996.

23. Goebl MG: The bmi-1 and mel-18 gene products define a new family of DNA-binding proteins involved in cell proliferation and tumorigenesis. Cell 66: 623, 1991.

24. Kanno M, Hasegawa M, Ishida $A$, Isono $K$ and Taniguchi $M$ : mel-18, a Polycomb group-related mammalian gene, encodes a transcriptional negative regulator with tumor suppressive activity. EMBO J 14: 5672-5678, 1995.

25. Moosmann P, Georgiev O, Le Douarin B, Bourquin JP and Schaffner W: Transcriptional repression by RING finger protein TIF1 beta that interacts with the KRAB repressor domain of KOX1. Nucleic Acids Res 24: 4859-4867, 1996.

26. Price BD, Chang Z, Smith R, Bockheim S and Laughon A: The Drosophila neutralized gene encodes a C3HC4 zinc finger. EMBO J 12: 2411-2418, 1993.

27. Satijn DP and Otte AP: RING1 interacts with multiple Polycombgroup proteins and displays tumorigenic activity. Mol Cell Biol 19: 57-68, 1999

28. Topcu Z, Mack DL, Hromas RA and Borden KL: The promyelocytic leukemia protein PML interacts with the proline-rich homeodomain protein PRH: a RING may link hematopoiesis and growth control. Oncogene 18: 7091-7100, 1999.

29. Satijn DP, Gunster MJ, van der Vlag J, Hamer KM, Schul W, Alkema MJ, Saurin AJ, Freemont PS, van Driel R and Otte AP: RING1 is associated with the polycomb group protein complex and acts as a transcriptional repressor. Mol Cell Biol 17: 4105-4113, 1997.

30. Obuse C, Yang H, Nozaki N, Goto S, Okazaki T and Yoda K: Proteomics analysis of the centromere complex from HeLa interphase cells: UV-damaged DNA binding protein 1 (DDB-1) is a component of the CEN-complex, while BMI-1 is transiently co-localized with the centromeric region in interphase. Genes Cells 9: 105-120, 2004.

31. Lee SJ, Choi D, Rhim H and Kang S: E3 ubiquitin ligase RNF2 interacts with the S6' proteasomal ATPase subunit and increases the ATP hydrolysis activity of S6'. Biochem J 389: 457-463, 2005.

32. Hanson PI and Whiteheart SW: $\mathrm{AAA}^{+}$proteins: have engine, will work. Nat Rev Mol Cell Biol 6: 519-529, 2005.

33. Lorick KL, Jensen JP, Fang S, Ong AM, Hatakeyama S and Weissman AM: RING fingers mediate ubiquitin-conjugating enzyme (E2)-dependent ubiquitination. Proc Natl Acad Sci USA 96: 11364-11369, 1999

34. Lee SJ, Choi JY, Sung YM, Park H, Rhim H and Kang S: E3 ligase activity of RING finger proteins that interact with Hip-2, a human ubiquitin-conjugating enzyme. FEBS Lett 503: 61-64, 2001 .

35. Ogretmen B and Safa AR: Expression of the mutated p53 tumor suppressor protein and its molecular and biochemical characterization in multidrug resistant MCF-7/Adr human breast cancer cells. Oncogene 14: 499-506, 1997.

36. Meek DW and Knippschild U: Posttranslational modification of MDM2. Mol Cancer Res 1: 1017-1026, 2003 